\title{
STEM Optical Sectioning for Imaging Screw Dislocations Core Structures in BCC Metals.
}

David Hernandez-Maldonado ${ }^{1,2}$, Hao Yang ${ }^{3}$, Lewys Jones ${ }^{2}$, Roman Gröger ${ }^{4}$, Peter B Hirsch ${ }^{2}$, Quentin M. Ramasse ${ }^{1}$ and Peter D Nellist ${ }^{1,2}$.

1. SuperSTEM Laboratory, SciTech Daresbury Campus, Warrington, UK

2. University of Oxford, Department of Materials, Parks Rd, Oxford, UK

${ }^{3 .}$ Lawrence Berkeley National Laboratory, California, United States

4. Institute of Physics of Materials ASCR and CEITEC IPM, Brno, Czech Republic

It is well known that the low-temperature plastic deformation of Body-Centred Cubic (BCC) metals is controlled by the glide of $1 / 2[111]$ screw dislocations. Their low mobility is caused by the delocalized nature of their cores [1] which are extended into several planes in the zone of the Burgers vector. This non-planar core spreading has been demonstrated by a number of atomistic studies made in the last 46 years [2]. However, attempts at experimental observation have been hindered by the Eshelby twist effect $[3,4]$. The aim of this work is to investigate whether the edge and screw displacements associated with 1/2[111] screw dislocations in BCC metals can be detected by optical sectioning in high-angle annular darkfield (HAADF) Scanning Transmission Electron Microscope (STEM) imaging conditions.

The introduction of spherical-aberration correctors in STEM has allowed an improvement in spatial resolution up to the sub-angstrom scale also accompanied by a reduction of the depth of focus (due to the increase in probe convergence angles), which in a modern instrument is just a few nanometers, thus often less than the sample thickness. This can be exploited to extract information along the beam direction by focusing the electron probe at specific depths within the sample. This technique has been employed in a recently published work with the aim to measure the dissociation distance of a mixed screw dislocation in $\mathrm{GaN}$ [5].

In this work we employ HAADF simulated images of a $1 / 2[111]$ screw dislocation in tungsten (W) to study the viability of the technique to resolve both components of the dislocation displacements. Screw displacements around the dislocation can be imaged for the dislocation lying in the plane perpendicular to the electron beam. Figure 1 shows a focal series of HAADF simulated images along [-101] extracted from a $10 \mathrm{~nm}$ thick model of $\mathrm{W}$ with a $1 / 2[111]$ screw dislocation placed in the centre of the crystal. It is possible to observe that as we focus the electron probe closer to where the screw dislocation is located, a shearing of the (-101) atomic planes becomes apparent. This is a tell-tale sign of the helical displacements that the atoms follow within the crystal.

In order to reveal the edge components of the dislocation we have created two atomistic models, one using the anisotropic linear-elastic displacements around the dislocation and the other using the core structure relaxed using the Bond Order Potential for W. Figures 2 a) and b) show the respective HAADF simulated images. Figure 2 c) is the RGB image made from the (101) component of the Fourier Transform (FT) (shown in Figure $2 \mathrm{~d}$ )) of both images. It is possible to observe that the shifts in this Fourier component occur along two distinct lines lying parallel to [111]. The superposition of both filtered images shows that there is a discrepancy on both sides of the core between both models. It is therefore apparent that the delocalisation of the core can in principle be detected using electron-optical sectioning [6,7]. 


\section{References}

[1] Hirsch, P. B. Fifth Internat. Congs. Crystallography, Cambridge, 139 (1960)

[2] Vitek, V. et al. Philosophical Magazine 21, 1049 (1970)

[3] Sigle, W. Philosophical Magazine 79, 1009 (1999)

[4] Mendis, B. G et al. Philosophical Magazine 86, 4607 (2006)

[5] Yang, H. et al. Nature Communications 6, 7266 (2015)

[6] Hernandez-Maldonado, D. et al. manuscript in preparation

[7] The SuperSTEM Laboratory is the U.K National Facility for Aberration-Corrected STEM, supported by the Engineering and Physical Science Research Council (EPSRC).
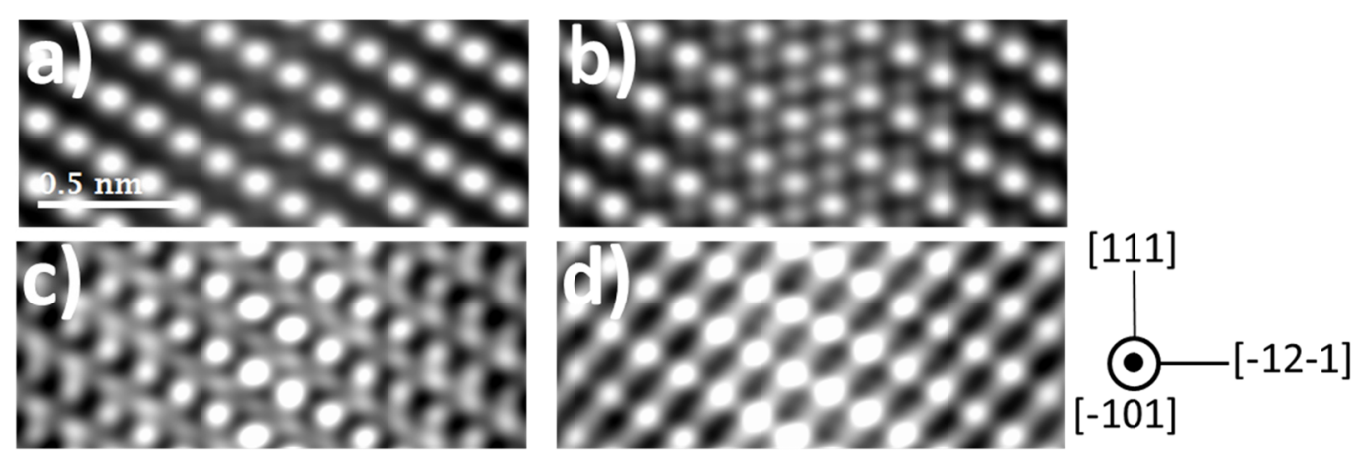

Figure 1. Simulated focal series of HAADF images of a screw dislocation viewed perpendicular to the dislocation line. The thickness of the model is $10 \mathrm{~nm}$ along the direction parallel to the electron beam and the dislocation is placed $5 \mathrm{~nm}$ from the entrance surface. The defocus value is a) $0 \mathrm{~nm}$, b) $3 \mathrm{~nm}$, c) $6 \mathrm{~nm}$ and d) $9 \mathrm{~nm}$. The maximum shearing has been found for a defocus of $6 \mathrm{~nm}$.
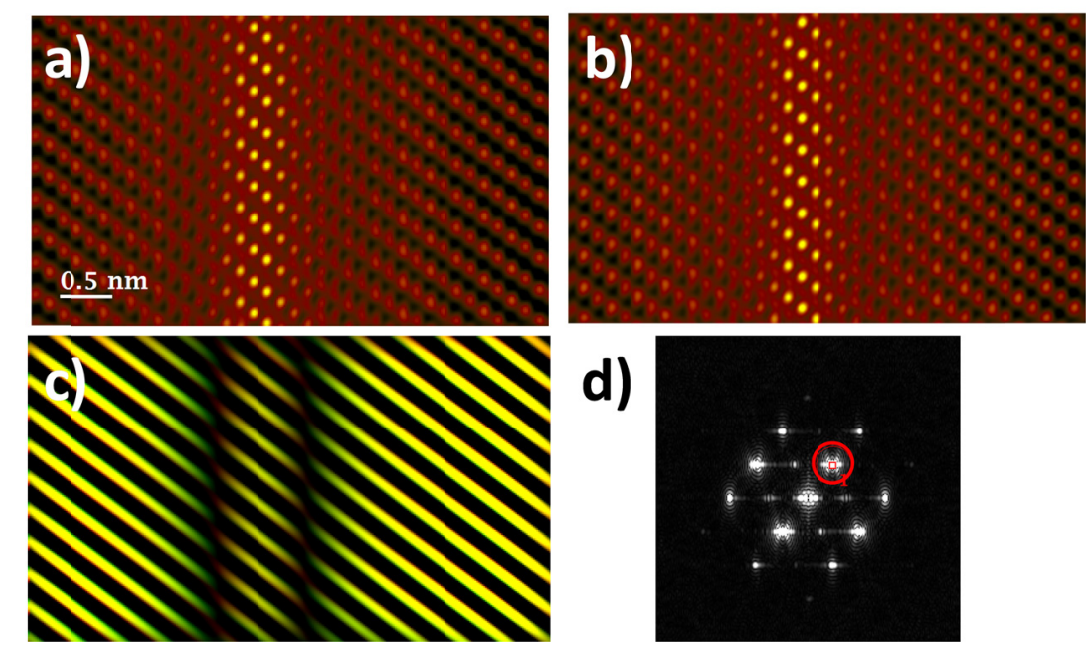

Figure 2. HAADF simulated images of a $1 / 2[111]$ screw dislocation for a defocus of $6 \mathrm{~nm}$ for: a) the atoms displaced by the anisotropic linear-elastic strain field of the dislocation, b) the dislocation core relaxed using molecular statics. c) Superposition of the inverse FT of the (101) component of both images. Red colour corresponds to Figure 2 a) Fourier filtered and green to Figure $2 \mathrm{~b}$ ). The places where the images coincide are in yellow. It is possible to observe how at both sides of the core there is a mismatch between the simulated images. d) FT of Figure $2 \mathrm{a}$ ), in red is marked the component used to compare images in c). 